\title{
Concentração de homocisteína em pacientes portadores de doença arterial periférica atendidos em um serviço público de saúde
}

\author{
Level of homocysteine in patients with peripheral arterial disease \\ treated at a public health care facility
}

Luciene de Souza Venâncio ${ }^{1}$, Roberto Carlos Burini ${ }^{2}$, Winston Bonetti Yoshida ${ }^{3}$

\begin{abstract}
Resumo
Contexto: Estudos recentes indicam que a concentração elevada de homocisteína é um fator de risco importante e prevalente para doença vascular aterosclerótica coronariana, cerebral e periférica.

Objetivo: Tendo em vista a escassez de informações relacionadas à hiper-homocisteinemia em doença arterial periférica (DAP) no Brasil e as peculiaridades de nossa população, o objetivo deste estudo foi avaliar a frequência de hiper-homocisteinemia em amostra dessa população em um ensaio clínico com indivíduos portadores e não portadores de DAP atendidos em um serviço público brasileiro.
\end{abstract}

Métodos: Foi realizado um estudo ensaio clínico caso-controle com 40 indivíduos portadores de DAP confirmada por Doppler ultrassom (grupo DAP) em comparação com 20 indivíduos voluntários sem DAP (grupo-controle).

Resultados: A DAP predominante foi a isquemia crônica de membros (75\%). As concentrações plasmáticas medianas de homocisteína de jejum foram significantemente maiores no grupo DAP do que no grupocontrole $(16,7$ versus $12,9 \mu \mathrm{mol} / \mathrm{L}, \mathrm{p}=0,001)$, tanto nos homens $(18,9$ versus $14,0 \mu \mathrm{mol} / \mathrm{L}, \mathrm{p}=0,005)$ quanto nas mulheres $(13,9$ versus $11,2 \mu \mathrm{mol} / \mathrm{L}, \mathrm{p}=0,025)$. Quanto à proporção de indivíduos com hiperhomocisteinemia, observou-se tendência a uma maior frequência no grupo DAP (60\%) em relação ao grupo-controle $(30 \%)(\mathrm{p}=0,054)$. Nos indivíduos com idade inferior a 60 anos foram encontrados valores medianos de homocisteína significantemente mais elevados no grupo $\operatorname{DAP}(\mathrm{p}=0,041)$.

Conclusões: A hiper-homocisteinemia é um fator de risco importante e foi encontrada em $60 \%$ dos indivíduos portadores de DAP atendidos em um serviço público no Brasil.

Palavras-chave: Homocisteína, hiper-homocisteinemia, doenças vasculares periféricas.

\begin{abstract}
Background: Recent studies have suggested that high level of homocysteine is an important and prevalent risk factor for coronary, cerebral and peripheral arterial disease.

Objective: In light of the lack of information on hyperhomocysteinemia in peripheral arterial disease (PAD) in Brazil and the peculiarities of its population, the objective of the present study was to evaluate the frequency of hyperhomocysteinemia in a sample of the Brazilian population by means of a clinical trial involving individuals with and without PAD being treated at a public health care facility.
\end{abstract}

Methods: A case-controlled clinical trial was conducted with 40 individuals with a PAD diagnosis confirmed by Doppler ultrasound (PAD group) compared with 20 volunteer individuals without PAD (control group).

Results: The predominant PAD was chronic limb ischemia (75\%). Median fasting plasma levels of homocysteine were significantly higher in the PAD group than in the control group (16.7 vs. $12.9 \mu \mathrm{mol} / \mathrm{L}$, $\mathrm{p}=0.001)$, both in men (18.9 vs. $14.0 \mu \mathrm{mol} / \mathrm{L}, \mathrm{p}=0.005)$ and women (13.9 vs. $11.2 \mu \mathrm{mol} / \mathrm{L}, \mathrm{p}=0.025)$. As to the proportion of individuals with hyperhomocysteinemia, a tendency toward a higher frequency was observed in the PAD group (60\%) in relation to the control group (30\%) $(p=0.054)$. Individuals aged less than 60 years had significantly high median values of homocysteine in the PAD group $(\mathrm{p}=0.041)$.

Conclusions: Hyperhomocysteinemia was a prevalent and important risk factor in individuals with PAD treated at a public health care facility in Brazil.

Keywords: Homocysteine, hyperhomocysteinemia, peripheral vascular diseases.

1. Nutricionista. Doutora, Bases Gerais da Cirurgia, Faculdade de Medicina de Botucatu, Universidade Estadual Paulista (UNESP), Botucatu, SP. Coordenadora, Curso de Graduação em Nutrição, Universidade Metodista de Piracicaba, Lins, SP.

2. Professor titular, Departamento de Saúde Pública. Coordenador, Centro de Metabolismo em Exercício e Nutrição, Faculdade de Medicina de Botucatu, UNESP, Botucatu, SP.

3. Professor adjunto, Departamento de Cirurgia e Ortopedia, Faculdade de Medicina de Botucatu, UNESP, Botucatu, SP.

Apoio financeiro concedido pela Coordenação de Aperfeiçoamento de Pessoal de Nível Superior (CAPES) e pela Fundação de Amparo à Pesquisa do Estado de São Paulo (FAPESP) (processo oㅜ 98/16098-8130).

Não foram declarados conflitos de interesse associados à publicação deste artigo.

Artigo submetido em 22.06.09, aceito em 02.10.09.

J Vasc Bras. 2009;8(4):318-326.

Copyright $\odot 2009$ by Sociedade Brasileira de Angiologia e de Cirurgia Vascular 


\section{Introdução}

Alguns fatores de risco para o desenvolvimento da doença aterosclerótica são bastante conhecidos, como idade, sexo masculino, dislipidemia, hábito tabágico, hipertensão aterial, diabetes melito, obesidade, sedentarismo e fatores genéticos ou história familiar de doença aterosclerótica ${ }^{1}$. Nos últimos anos, foram identificados outros fatores de risco, como a homocisteína, cujo estudo pode ampliar o entendimento sobre os mecanismos fisiopatológicos da aterosclerose e possibilitar o desenvolvimento de novas medidas preventivas ou terapêuticas.

A homocisteína é um aminoácido sulfurado sintetizado em condições fisiológicas a partir da metionina alimentar, sendo utilizado em diversas vias metabólicas. A hiper-homocisteinemia pode ser atribuída à ocorrência de defeitos genéticos de algumas enzimas do metabolismo da homocisteína, ou a deficiências nutricionais, ou, ainda, estar relacionada a outros fatores de risco para aterosclerose. Dentre as causas não genéticas de hiper-homocisteinemia, o estado nutricional parece ser o parâmetro mais importante na regulação da concentração da homocisteína. Merecem destaque as alterações nutricionais relacionadas a deficiências das vitaminas B12, B6 e, principalmente, folato, que são cofatores no metabolismo da homocisteína ${ }^{2}$.

Essas deficiências são altamente prevalentes e podem provocar hiper-homocisteinemia moderada, sendo a concentração plasmática de homocisteína inversamente proporcional aos níveis sanguíneos de folato, vitamina B6 e B12 e à ingestão dessas vitaminas ${ }^{3,4}$. Inúmeras pesquisas epidemiológicas têm demonstrado que a elevação das concentrações sanguíneas de homocisteína pode ser um fator de risco adicional para doença vascular coronariana ${ }^{5}$, cerebral $^{6}$ ou periférica ${ }^{7}$, bem como para trombose venosa ${ }^{8}$. A hiper-homocisteinemia foi detectada em 28 a $30 \%$ dos pacientes com doença arterial periférica (DAP) ${ }^{7}$, e as concentrações médias de homocisteína em pacientes com diversas manifestações de DAP mostraram-se significantemente superiores às encontradas nos controles, na claudicação intermitente ${ }^{9}$, nas lesões íleo-femorais ${ }^{10}$, na síndrome de Leriche ${ }^{11}$, na estenose de carótida ${ }^{12}$ e no aneurisma de aorta abdominal ${ }^{13}$.

Estudos epidemiológicos no Brasil revelaram prevalência alta de $20 \%$ de hiper-homocisteinemia em indivíduos nipo-brasileiros portadores de arteriopatia periférica aterosclerótica, com valores médios de homocisteína no sexo masculino progressivamente mais elevados conforme a gravidade do estado glicêmico ${ }^{14,15}$. A importância da hiper-homocisteinemia como fator de risco adicional para a DAP é bastante conhecida, com uma razão de chance (odds ratio, OR) de $6,8^{3}$ até $11^{16}$ para o seu desenvolvimento. Além disso, a hiper-homocisteinemia está associada ao aumento da progressão da $\mathrm{DAP}^{17,18}$ e do risco de morte precoce por doença cardiovascular ${ }^{4}$ e doença arterial coronariana em pacientes com DAP sintomática ${ }^{17,19}$. Com base nesses estudos, a homocisteína sanguínea parece ser um importante marcador preditor de DAP, principalmente em indivíduos com aterosclerose prematura ou história familiar grave de aterotrombose e na ausência de outros fatores de risco ${ }^{20}$.

Foram sugeridos vários mecanismos fisiopatológicos para lesão vascular causada pelo aumento da homocisteína no plasma, incluindo agressão direta ao endotélio, indução do estresse oxidativo na parede vascular e oxidação de lipoproteínas, alteração na produção de óxido nítrico e alterações das propriedades antitrombóticas ${ }^{21}$.

Tendo em vista a escassez de informações nutricionais relacionadas com a hiper-homocisteinemia em DAP no Brasil e as peculiaridades da nossa população mais carente, usuária do Sistema Único de Saúde (SUS), o objetivo deste estudo foi avaliar a frequência de hiper-homocisteinemia em amostra dessa população em um estudo caso-controle com 40 pacientes portadores de DAP confirmada por Doppler ultrassom em comparação com 20 indivíduos voluntários sem DAP (controles).

\section{Métodos}

O estudo foi aprovado pela Comissão de Ética em Pesquisa (protocolo $\mathrm{n}^{\mathrm{o}}$ 087/99). Todos os sujeitos que participaram do presente estudo foram informados em detalhes sobre os procedimentos a que seriam submetidos e assinaram o termo de consentimento livre e esclarecido. Foi realizado um estudo caso-controle no qual o grupo de estudo foi composto por 40 indivíduos com DAP sintomática (grupo DAP), que chegaram consecutivamente ao ambulatório do Serviço de Cirurgia Vascular entre março de 1999 e outubro de 2001 com graus de isquemia de I a III segundo Rutherford et al. ${ }^{22}$ e índice de pressão tornozelo-braço (IPTB) igual ou inferior a $0,90^{23}$. Não foram incluídos neste grupo indivíduos com cirurgia importante planejada durante o período do estudo, portadores de arterites e am- 
putações prévias, com enfermidade hepática ou renal conhecida clinicamente, em gestação, com diagnóstico de HIV, câncer, ou enfermidades com proliferação celular, insuficiência cardíaca e/ou respiratória, recebendo alimentação artificial, via parenteral ou enteral, em uso crônico de suplementos de ácido fólico, polivitamínicos e/ou de medicamentos que pudesse interferir no metabolismo da homocisteína. No grupo-controle foram incluídos 20 indivíduos saudáveis sem DAP confirmada objetivamente e com IPTB maior que 0,90, procedentes da mesma região geográfica e atendidos no mesmo hospital, sendo recrutados de março de 1999 a dezembro de 2000.

Foram investigados os seguintes fatores de risco para doença aterosclerótica:

1) hábito tabágico pregresso e atual;

2) excesso de peso: índice de massa corporal maior que $24,9 \mathrm{~kg} / \mathrm{m}^{2}$, segundo a Organização Mundial da Saút $\mathrm{de}^{24}$;

3) excesso de adiposidade abdominal: circunferência da cintura maior que $88 \mathrm{~cm}$ para mulheres e $102 \mathrm{~cm}$ para homens $^{25}$;

4) dislipidemia: colesterol total maior que $200 \mathrm{mg} / \mathrm{dL}$, lipoproteína de alta densidade (HDL-C) menor que $45 \mathrm{mg} / \mathrm{dL}$ e triacilglicerol maior que $150 \mathrm{mg} / \mathrm{dL}^{25}$, pelo método enzimático colorimétrico automatizado em aparelho RA-XT Technicon-EUA, e lipoproteína de baixa densidade (LDL-C) maior que $100 \mathrm{mg} / \mathrm{dL}^{25}$, calculada segundo a fórmula de Friedewald;

5) doenças com comprovação clínica e laboratorial documentadas no prontuário médico, como hipertensão arterial e diabetes melito.

As análises das concentrações de homocisteína plasmática total de jejum foram realizadas por cromatografia líquida de alta precisão (high performance liquid chromatography - HPLC) da Shimadzu (LC-10AD), através de derivatização pré-coluna com SBD-F e posterior detecção por fluorescência, segundo padronização proposta por Araki \& Sako ${ }^{26}$ modificada por Ubbink et al. ${ }^{27}$. Os níveis normais de homocisteína total foram definidos entre 5 e $15 \mu \mathrm{mol} / \mathrm{L}$, conforme Ueland et al. ${ }^{28}$. A colheita de sangue em veia periférica foi realizada após período de repouso e jejum de 8 a 12 horas.
Empregou-se, para as análises estatísticas, o pacote estatístico Sigma Stat for Windows, versão 3.5. Foram empregadas estatísticas descritivas como a mediana e intervalos interquartis, sendo que as medianas de todas as variáveis foram comparadas entre o grupo-controle e o grupo DAP através do teste $t$ não pareado (Mann-Whitney) e qui-quadrado (ou teste exato de Fisher), sendo as diferenças consideradas significantes quando $\mathrm{p} \leq 0,05$. Calculou-se ainda a OR para predição do risco de indivíduos com hiper-homocisteinemia do grupo-controle em desenvolver DAP.

\section{Resultados}

A Tabela 1 exibe as características comparativas entre os dois grupos estudados. Os indivíduos do grupo-controle, em relação aos do grupo DAP, apresentaram valores significantemente inferiores quanto a idade, circunferência da cintura, creatinina sérica, colesterol total sérico, LDL-C e triacilglicerol, e valores superiores quanto ao IPTB, HDL-C e presença de hipertensão arterial e diabetes melito. Notou-se maior frequência $(75 \%)$ de indivíduos com isquemia crônica de membros em relação a outras enfermidades arteriais $(\mathrm{p}=0,05)$ (Tabela 2$)$.

As medianas das concentrações plasmáticas de homocisteína de jejum nos indivíduos do grupo-controle foram normais $(12,9 \mu \mathrm{mol} / \mathrm{L})$ e significantemente inferiores aos do grupo DAP $(16,7 \mu \mathrm{mol} / \mathrm{L})(\mathrm{p}=0,001)$ (Figura 1). Os homens $(p=0,005)$ e as mulheres $(p=0,025)$ do grupo DAP apresentaram valores significantemente mais elevados de homocisteína quando comparados aos valores do grupo-controle (Figura 2). Considerando-se a classificação da hiper-homocisteinemia quanto à sua forma, 30\% dos indivíduos do grupo-controle apresentaram hiperhomocisteinemia leve, definida como valores plasmáticos de até $30 \mu \mathrm{mol} / \mathrm{L}$; no grupo DAP, a forma leve de hiper-homocisteinemia foi predominante $(50 \%)$. Dez por cento dos indivíduos com DAP apresentaram hiper-homocisteinemia moderada (31 a $100 \mu \mathrm{mol} / \mathrm{L}$ ) (Figura 3). Portanto, quanto à proporção de indivíduos com hiper-homocisteinemia, observou-se tendência a uma maior frequência no grupo DAP em relação ao grupo-controle $(\mathrm{p}=0,054)$.

Considerando-se a idade, os grupos foram classificados em idade menor e maior ou igual a 60 anos. Nos indivíduos com idade inferior a 60 anos foram encontrados 
Tabela 1 - Distribuição da frequência e valores medianos dos dados demográficos e clínico-nutricionais dos grupos estudados: grupo-controle (sem doença arterial periférica) e grupo DAP (com doença arterial periférica)

\begin{tabular}{|c|c|c|c|}
\hline Características & Grupo-controle $(\mathrm{n}=20)$ & Grupo DAP $(\mathrm{n}=40)$ & $\mathrm{p}$ \\
\hline \multicolumn{4}{|l|}{ Sexo, n (\%) } \\
\hline Masculino & $10(50)$ & $24(60)$ & \multirow{2}{*}{$\chi^{2}=0,790$} \\
\hline Feminino & $10(50)$ & $16(40)$ & \\
\hline \multicolumn{4}{|l|}{ Idade (anos) } \\
\hline Mediana & 35 & 61 & \multirow[t]{2}{*}{$\mathrm{MW}<0,001$} \\
\hline Percentis 25 e 75 & $31-38$ & $51-70$ & \\
\hline \multicolumn{4}{|l|}{ Raça, n (\%) } \\
\hline Branca & $18(90)$ & $37(92,5)$ & \multirow[t]{2}{*}{$\mathrm{TEF}=1,000$} \\
\hline Não branca & $2(10)$ & $3(7,5)$ & \\
\hline \multicolumn{4}{|l|}{ Ocupação, n (\%) } \\
\hline Aposentados & $2(10)$ & $24(60)$ & \multirow[t]{2}{*}{$\mathrm{TEF}<0,001 *$} \\
\hline Ativos & $18(90)$ & $16(40)$ & \\
\hline \multicolumn{4}{|l|}{ Procedência, n (\%) } \\
\hline Botucatu & $16(80)$ & $13(32,5)$ & \multirow[t]{2}{*}{$\chi^{2}=0,001 *$} \\
\hline Outras cidades & $4(20)$ & $27(67,5)$ & \\
\hline \multicolumn{4}{|l|}{ IPTB } \\
\hline Mediana & 1,0 & 0,60 & \multirow{2}{*}{$\mathrm{MW}<0,001 *$} \\
\hline Percentis 25 e 75 & $0,95-1,14$ & $0,47-0,80$ & \\
\hline \multicolumn{4}{|l|}{ Grau de isquemia, $\mathrm{n}(\%)$} \\
\hline I & - & $25(62,5)$ & \\
\hline II & - & $3(7,5)$ & \\
\hline III & - & $9(22,5)$ & - \\
\hline \multicolumn{4}{|l|}{$\operatorname{IMC}\left(\mathrm{kg} / \mathrm{m}^{2}\right)$} \\
\hline Mediana & 25,6 & 25,2 & \multirow[t]{2}{*}{$\mathrm{MW}=0,410$} \\
\hline Percentis 25 e 75 & $21,8-27,5$ & $22,9-28,9$ & \\
\hline \multicolumn{4}{|l|}{$\mathrm{CC}(\mathrm{cm})$} \\
\hline Mediana & 88,5 & 93 & \multirow[t]{2}{*}{$\mathrm{MW}=0,006^{*}$} \\
\hline Percentis 25 e 75 & $75-94,7$ & $89-100$ & \\
\hline \multicolumn{4}{|l|}{ Creatinina (mg/\%) } \\
\hline Mediana & 0,95 & 1,0 & \multirow[t]{2}{*}{$\mathrm{MW}=0,032^{*}$} \\
\hline Percentis 25 e 75 & $0,85-1,0$ & $0,9-1,3$ & \\
\hline \multicolumn{4}{|l|}{ Colesterol total (mg/\%) } \\
\hline Mediana & 184 & 214 & \multirow[t]{2}{*}{$\mathrm{MW}=0,007 *$} \\
\hline Percentis 25 e 75 & $162-212$ & $185-262$ & \\
\hline \multicolumn{4}{|l|}{ LDL-C (mg/\%) } \\
\hline Mediana & 124 & 139,5 & \multirow[t]{2}{*}{$\mathrm{MW}=0,018^{*}$} \\
\hline Percentis 25 e 75 & $81,5-145$ & $117-179$ & \\
\hline HDL-C (mg/\%) & & & \\
\hline Mediana & 42,5 & 37,5 & $\mathrm{MW}=0,121$ \\
\hline Percentis 25 e 75 & $35,5-54$ & $30,5-46$ & \\
\hline Triacilglicerol (mg/\%) & & & \\
\hline Mediana & 119,5 & 155,5 & $\mathrm{MW}=0,019^{*}$ \\
\hline Percentis 25 e 75 & $75,5-144,5$ & $98,5-225,5$ & \\
\hline Hábito tabágico, n (\%) & & & \\
\hline Não fumantes & $1(5)$ & $21(52,5)$ & $\mathrm{TEF}=1,000$ \\
\hline Ex-fumantes e fumantes & - & $15(37,5)$ & \\
\hline Hipertensão arterial, n (\%) & $3(15)$ & $26(65)$ & - \\
\hline Diabetes melito, n (\%) & - & $8(20)$ & - \\
\hline
\end{tabular}

$\%=$ porcentagem absoluta (em relação ao número total de indivíduos em cada grupo); $\chi^{2}=$ teste do qui-quadrado; $\mathrm{CC}=$ circunferência da cintura; DAP = doença arterial periférica; HDL-C = lipoproteína de alta densidade; IMC = índice de massa corporal; IPTB = índice de pressão tornozelo-braço; LDL-C = lipoproteína de baixa densidade; MW = Mann-Whitney; $\mathrm{n}=$ total de pacientes; TEF = teste exato de Fisher.

* Valor de $\mathrm{p}$ indica diferença estatística significante entre os grupos. 
Tabela 2 - Distribuição da frequência do tipo de doença arterial periférica no grupo DAP

\begin{tabular}{lc}
\hline Tipo de DAP & $\mathrm{n}(\%)$ \\
\hline Isquemia crônica de membros & $30(75)$ \\
Síndrome de Leriche & $7(17,5)$ \\
Oclusão arterial aguda & $1(2,5)$ \\
Aneurisma de aorta abdominal & $1(2,5)$ \\
Aneurisma de artéria poplítea & $1(2,5)$ \\
\hline
\end{tabular}

$\mathrm{DAP}=$ doença arterial periférica.

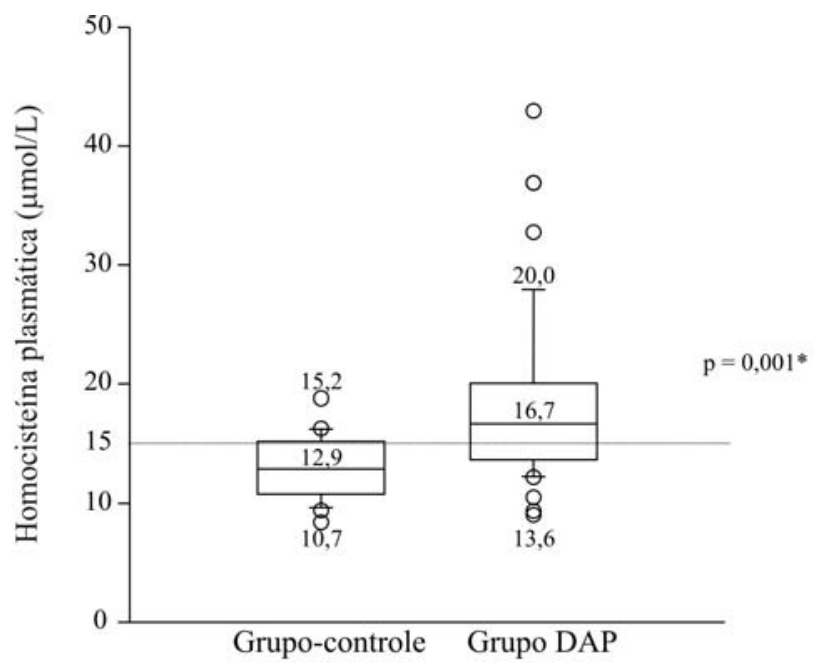

Os valores são apresentados na forma de medianas e percentis 25 e 75 . Teste estatistico: Mann-Whitney.

$\mathrm{DAP}=$ doença arterial periférica.

* Valor de p indica diferença estatística significante entre os grupos.

Figura 1 - Medianas e variação da concentração de homocisteína plasmática de jejum dos grupos estudados

valores medianos de homocisteína significantemente mais elevados no grupo DAP: grupo DAP $14,9 \mu \mathrm{mol} / \mathrm{L}(12,5-$ $18,1)$ versus grupo-controle $12,8 \mu \mathrm{mol} / \mathrm{L}(10,5-15,2)$, $\mathrm{p}=0,041$.

O cálculo da OR identificou que indivíduos saudáveis com homocisteína superior a $15 \mu \mathrm{mol} / \mathrm{L}$ têm 3,5 vezes mais chances de desenvolver DAP do que indivíduos com homocisteína inferior a $15 \mu \mathrm{mol} / \mathrm{L}$ (OR 3,5; intervalo de confiança de 95\%: 1,45-8,57; $\mathrm{p}=0,003)$.

\section{Discussão}

Esta investigação com indivíduos portadores de DAP usuários do sistema de saúde pública do Brasil (SUS) é importante uma vez que, em sua maioria, eles possuem con-
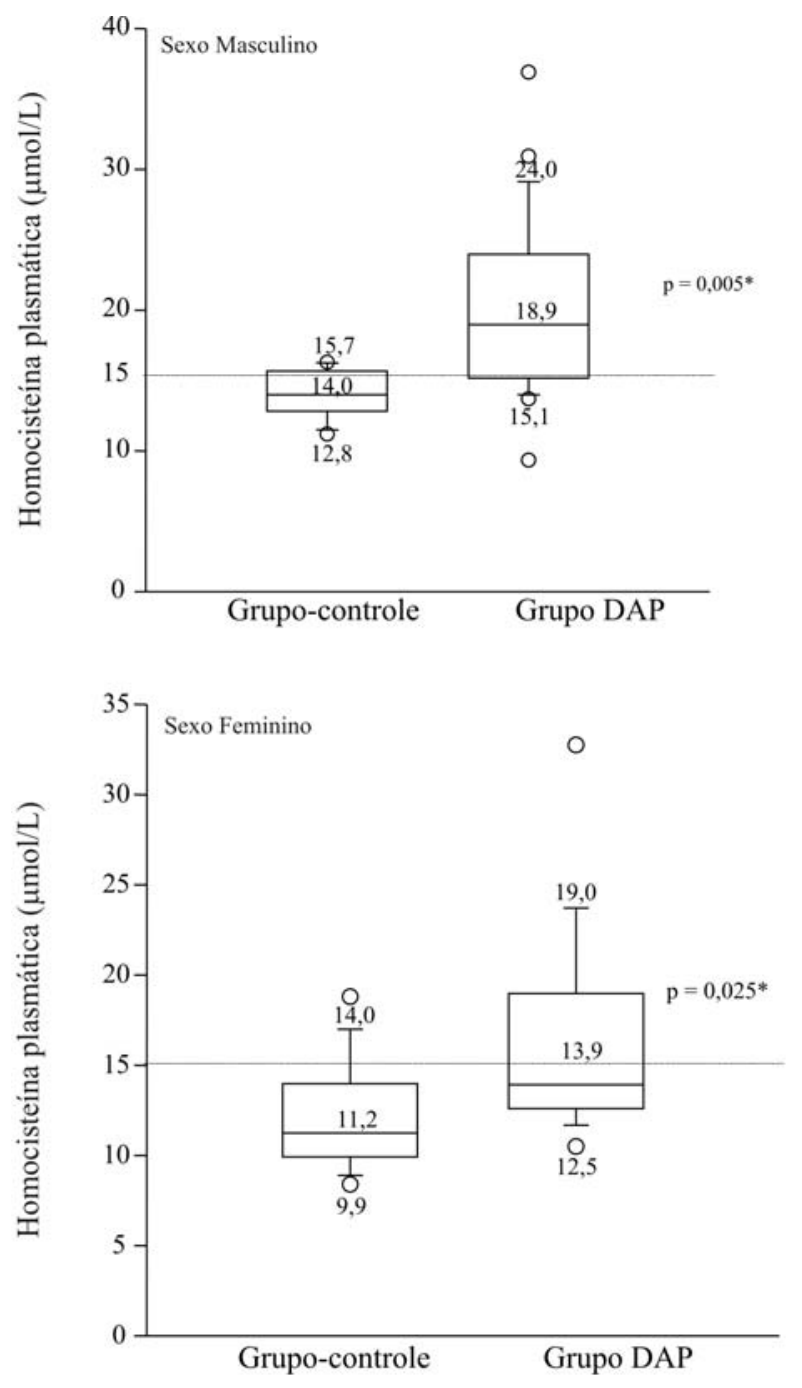

Os valores são apresentados na forma de medianas e percentis 25 e 75 . Teste estatístico: Mann-Whitney.

$\mathrm{DAP}=$ doença arterial periférica

* Valor de p indica diferença estatistica significante entre os grupos.

Figura 2 - Medianas e variação da concentração de homocisteína plasmática de jejum dos grupos estudados de acordo com o sexo

dições peculiares socioeconômicas e de escolaridade e hábitos alimentares e de vida próprios da região onde residem e, portanto, poderiam apresentar um comportamento distinto de outros contingentes nacionais e internacionais quanto aos fatores de risco para aterosclerose relacionada como a homocisteína.

A importância da hiper-homocisteinemia como marcador de risco para a DAP é bastante conhecida. Concentrações plasmáticas de homocisteína acima de $10 \mu \mathrm{mol} / \mathrm{L}$, ou um aumento de $5 \mu \mathrm{mol} / \mathrm{L}$, aumentam em 6,8 o risco para o desenvolvimento de $\mathrm{DAP}^{3,29}$. Além disso, a hi- 


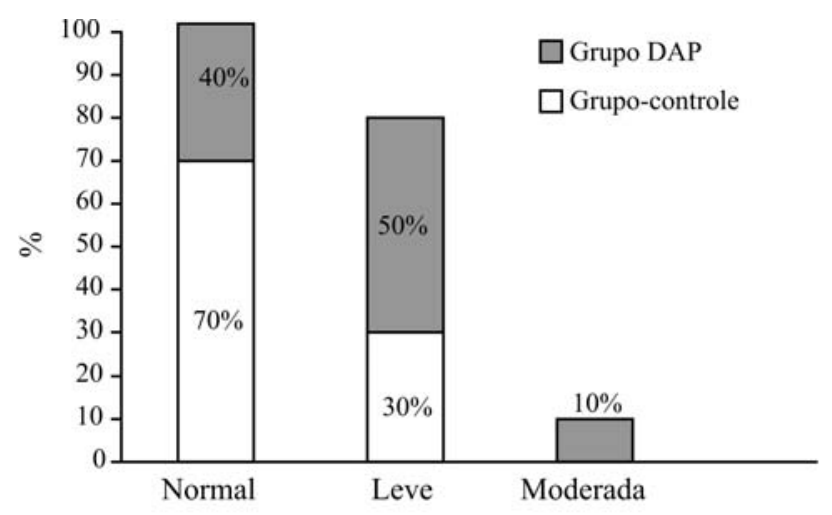

Concentração de homocisteina: normal $=5$ a $15 \mu \mathrm{mol} / \mathrm{L}$; leve $=16$ a $30 \mu \mathrm{mol} / \mathrm{L}$; moderada $=31$ a $100 \mu \mathrm{mol} / \mathrm{L}$.

Teste estatistico: teste exato de Fisher.

$\mathrm{DAP}=$ doença arterial periférica.

* Valor de p indica diferença estatística significante entre os grupos.

Figura 3 - Distribuição da frequência da classificação de hiper-homocisteinemia nos grupos estudados

per-homocisteinemia está associada ao aumento de risco de morte por doença cardiovascular ${ }^{4}$ e progressão da doença arterial coronariana em pacientes com DAP sintomáti$\mathrm{ca}^{17,18}$. A análise clínica da homocisteína sanguínea como um marcador preditor de DAP é considerada de grande importância, principalmente em indivíduos com aterosclerose prematura ou com história familiar grave de aterotrombose e com ausência de outros fatores de risco $^{20}$. Entretanto, os resultados de outros estudos epidemiológicos prospectivos realizados em populações sem história pregressa de doença cardiovascular mostraram-se conflitantes quanto à presença de elevada concentração sanguínea de homocisteína como fator de risco para mortalidade por doença arterial coronariana, após alguns anos de seguimento $^{30}$.

Como no presente estudo, outros foram realizados em pacientes portadores de DAP sintomática (manifestada por claudicação intermitente, estenose de carótida, aneurisma de aorta abdominal, lesão íleo-femoral e aorto-ilía$\mathrm{ca}^{31,32}$ ), demonstrando que a concentração sanguínea de homocisteína, bem como a prevalência de hiper-homocisteinemia (20 a 78\%) eram significantemente maiores nos pacientes do que nos controles, mesmo após ajuste estatístico para outros fatores de risco para doenças vasculares. No presente estudo, a hiper-homocisteinemia foi encontrada em $60 \%$ dos pacientes com DAP confirmada, com predominância da forma moderada. Outros estudos nacionais também revelaram prevalência alta de hiperhomocisteinemia em indivíduos portadores de aneurisma de aorta abdominal (11\%) e doença obstrutiva da aorta abdominal $(33 \%)^{33}$. Em indivíduos nipo-brasileiros portadores de arteriopatia periférica aterosclerótica, as concentrações médias de homocisteína foram progressivamente mais elevadas conforme a gravidade do estado glicêmico ${ }^{14}$ e no sexo masculino ${ }^{15,34}$. Além disso, a concentração de homocisteína estaria relacionada com a idade e o sexo. Muito embora o grupo-controle tenha apresentado valores medianos de idade inferiores em relação ao grupo DAP, os resultados do presente estudo vêm de encontro aos de outros $^{35,36}$ que também observaram níveis mais elevados de homocisteína nos homens do que nas mulheres e aumento progressivo com a idade. Avaliando as diferenças existentes no ciclo da metionina com isótopos estáveis em homens e mulheres, Fukagawa et al. ${ }^{37}$ observaram que a taxa de transmetilação e remetilação da homocisteína era maior nas mulheres do que nos homens. Os autores sugeriram que a diferença existente entre homens e mulheres quanto à necessidade e a utilização de certos aminoácidos seria responsável pelo aumento da remetilação nas mulheres. Além disso, outros autores sugeriram que o metabolismo da homocisteína seria influenciado por hormônios sexuais femininos, como o estrógeno; entretanto, os mecanismos pelos quais o estrógeno provocaria redução nos níveis de homocisteína ainda são desconhecidos ${ }^{38}$. A diminuição da produção ou da atividade enzimática do metabolismo da homocisteína, o declínio fisiológico na função renal e a diminuição da biodisponibilidade de vitaminas como folato, B6 e B12 poderiam explicar o aumento nas concentrações de homocisteína com a idade ${ }^{3,36}$. De acordo com Hernanz ${ }^{39}$, esta elevação da homocisteína com a idade afetaria negativamente o estado pró-oxidante das células por aumentar os níveis de espécies reativas de oxigênio e, consequentemente, favorecer a propensão de maior risco de doenças crônicas não transmissíveis, em especial aquelas associadas ao endotélio.

Vários estudos têm mostrado a relação entre composição corporal e níveis de homocisteína. $\mathrm{O}$ indicador da composição corporal utilizado nestes estudos foi o índice de massa corporal (IMC), o qual se correlacionou positiva e significantemente com os níveis de homocisteína, especialmente em indivíduos obesos ${ }^{35}$ e particularmente naqueles com IMC igual ou maior que $30,7 \mathrm{~kg} / \mathrm{m}^{2}$, segundo Jacques et al. ${ }^{36}$. Nos pacientes do presente estudo, o IMC e a circunferência da cintura mostraram-se significantemen- 
te elevados no grupo DAP em relação ao grupo-controle, e grande parte dos hiper-homocisteinêmicos tinha peso corporal excessivo e aumento de gordura corporal e abdominal, especialmente entre as mulheres. Este padrão de composição corporal, associado à presença de níveis aumentados de homocisteína, contribuiria para um risco maior de desenvolvimento de doença aterosclerótica ${ }^{35}$.

Alguns autores observaram uma correlação positiva significante entre níveis de homocisteína, colesterol total e triacilglicerol ${ }^{35}$. Além disso, concentrações elevadas de homocisteína em pacientes com DAP resultaram em um aumento de duas vezes no risco de DAP em pacientes hipercolesterolêmicos (risco relativo observado 2,1) ${ }^{4}$. É importante salientar a possível interação entre a lipoproteína de baixa densidade (LDL) e a homocisteína no mecanismo fisiopatológico da aterosclerose, uma vez que o grupo de pacientes com DAP do presente estudo manifestou hipertrigliceridemia, hipercolesterolemia e aumento de LDL.

Portanto, a relação entre a elevação da concentração plasmática de homocisteína e o risco de doenças ateroscleróticas, em especial a DAP, já está bem estabelecida. Adicionalmente, a hiper-homocisteinemia aumentaria o risco de doença aterosclerótica quando em combinação com outros fatores que provocariam lesões vasculares.

Embora a elevação do aminoácido homocisteína no sangue esteja associada a disfunção endotelial, trombose e maior gravidade da aterosclerose, ainda não há consenso sobre as possibilidades terapêuticas. Uma meta-análise realizada por Clarke et al. ${ }^{40}$ indicou que dentre as vitaminas estudadas (B6, B12 e ácido fólico), o ácido fólico promoveu uma redução de até $25 \%$ na homocisteína sanguínea quando suplementado de 50 a $500 \mu \mathrm{g}$ diariamente. Outra meta-análise realizada por Boushey et al. ${ }^{3}$ indicou que um aumento diário de $200 \mu \mathrm{g}$ na ingestão de folato dietético levaria a uma redução de $4 \mu \mathrm{mol} / \mathrm{L}$ nas concentrações de homocisteína plasmática de jejum em pacientes portadores de doença cardiovascular, cerebrovascular e arterial periférica. Outros autores consideraram ainda a suplementação vitamínica com ácido fólico benéfica e não onerosa, além de poder ser adotada como uma terapia adjuvante em pacientes em doença aterosclerótica sintomática ou não, bem como na prevenção do aparecimento da $\mathrm{DAP}^{41}$.

Em resumo, os pacientes com DAP participantes deste estudo foram em geral indivíduos idosos, homens, predo- minantemente portadores de insuficiência arterial periférica, tanto nas formas crônicas como nas agudas. Os fatores de risco associados para aterosclerose foram hipertensão arterial sistêmica, diabetes melito, hábito tabágico e obesidade, alterações no metabolismo de lipídios e concentrações elevadas de homocisteína plasmática. Esses achados enfatizam o interesse e a importância do estudo da homocisteína em pacientes com DAP pelo envolvimento de fatores nutricionais no risco de desenvolvimento e na terapêutica de eventos aterotrombóticos.

\section{Conclusão}

A hiper-homocisteinemia é um fator de risco importante e foi encontrada em $60 \%$ dos indivíduos portadores de DAP atendidos em um serviço público no Brasil. Torna-se relevante a realização de estudos clínicos controlados e prospectivos nesses casos em particular para examinar os efeitos precisos da redução da homocisteína sanguínea quanto ao risco, à incidência e à história natural da doença aterosclerótica a fim de prevenir a progressão ou possibilitar a regressão das lesões ateroscleróticas periféricas.

\section{Agradecimentos}

À biomédica Maria Dorotéia Borges dos Santos pela dosagem da homocisteína; aos responsáveis clínicos pelos pacientes (Prof. Dr. Francisco H. A. Maffei, Prof. Dr. Sidnei Lastória, Prof. Dr. Hamilton A. Rollo, Profa. Dra. Regina Moura, Profa. Dra. Mariangela Giannini); ao matemático Prof. Dr. Adalberto Crocci (in memoriam) pela assistência na análise estatística e aos funcionários do Serviço de Cirurgia Vascular do Laboratório de Análises Clínicas e do Centro de Metabolismo em Exercício e Nutrição do Hospital das Clínicas da Faculdade de Medicina de Botucatu - UNESP.

\section{Referências}

1. Bhatt DL, Steg PG, Ohman EM, et al. International prevalence, recognition, and treatment of cardiovascular risk factors in outpatients with atherothrombosis. JAMA. 2006;295:180-9.

2. Selhub J. Homocysteine metabolism. Annu Rev Nutr. 1999;19:217-46.

3. Boushey CJ, Beresford SA, Omenn GS, Motulsky AG. A quantitative assessment of plasma homocysteine as a risk factor for vascular disease. JAMA. 1995;274:1049-57.

4. Graham IM, Daly LE, Refsum HM, et al. Plasma homocysteine as a risk factor for vascular disease. The European Concerted Action Project. JAMA. 1997;277:1775-81. 
5. Arnesen E, Refsum HM, Bonaa KH, Ueland PM, Forde OH, Nordrehaug JE. Serum total homocysteine and coronary heart disease. Int J Epidemiol. 1995;24:704-9.

6. Verhoef P, Hennekens CH, Malinow MR, Kok FJ, Willett WC, Stampfer MJ. A prospective study of plasma homocyst(e)ine and risk of ischemic stroke. Stroke. 1994;25:1924-30.

7. Boers GH, Smals AG, Trijbels FJ, et al. Heterozygosity for homocystinuria in premature peripheral and cerebral occlusive arterial disease. N Engl J Med. 1985;313:709-15.

8. Morelli VM, Lourenço DM, D'Almeida V, et al. Hyperhomocysteinemia increases the risk of venous thrombosis independent of the C677T mutation of the methylenetetrahydrofolate reductase gene in selected Brazilian patients. Blood Coagul Fibrinolysis. 2002;13:271-5.

9. Mölgaard J, Malinow MR, Lassvik C, Holm AC, Upson B, Olsson AG. Hyperhomocyst(e)inaemia: an independent risk factor for intermittent claudication. J Intern Med. 1992;231:273-9.

10. Malinow MR, Kang SS, Taylor LM, et al. Prevalence of hyperhomocyst(e)inemia in patients with peripheral arterial occlusive disease. Circulation. 1989;79:1180-8.

11. Brattström L, Israelson B, Norrving B, et al. Impaired homocysteine metabolism in early-onset cerebral and peripheral occlusive arterial disease. Atherosclerosis. 1990;81:51-60.

12. Selhub J, Jacques PF, Bostom AG, et al. Association between plasma homocysteine concentrations and extracranial carotid-artery stenosis. N Engl J Med. 1995;332:286-91.

13. Brunelli T, Prisco D, Fedi S, et al. High prevalence of mild hyperhomocysteinemia in patients with abdominal aortic aneurysm. J Vasc Surg. 2000;32:531-6.

14. Garófolo L, Barros Jr N, Ferreira SR, Sanudo A, Miranda Jr F. Moderate hyperhomocysteinemia in atherosclerotic peripheral arterial disease in the diabetics Nipo-Brazilian population in the city of Bauru. J Vasc Bras. 2003;2(suppl 1):S82.

15. Miranda Jr F, Garófolo L, Barros Jr N, Ferreira SR, Sanudo A. Influência do gênero na associação entre a hiperhomocisteinemia moderada e arteriopatia periférica em nipo-brasileiros de Bauru. J Vasc Bras. 2003;2:S82.

16. Kuan YM, Dear AE, Grigg MJ. Homocysteine: an etiological contributor to peripheral vascular arterial disease. ANZ J Surg. 2002;72:668-71.

17. Nicoloff AD, Taylor LM, Sexton GJ, et al. Relationship between site of initial symptoms and subsequent progression of disease in a prospective study of atherosclerosis progression in patients receiving long-term treatment for symptomatic peripheral arterial disease. J Vasc Surg. 2002;35:38-47.

18. Taylor LM, De Frang RD, Harris Jr EJ, Porter JM. The association of elevated plasma homocyst(e)ine with progression of symptomatic peripheral arterial disease. J Vasc Surg. 1991;13:128-36.

19. Taylor LM, Moneta GL, Sexton GJ, Schuff RA, Porter JM. Prospective blinded study of the relationship between plasma homocysteine and progression of symptomatic peripheral arterial disease. J Vasc Surg. 1999;29:8-21.

20. Ridker PM, Stampfer MJ, Rifai N. Novel risk factors for systemic atherosclerosis: a comparison of C-reactive protein, fibrinogen, homocysteine, lipoprotein(a), and standard cho- lesterol screening as predictors of peripheral arterial disease. JAMA. 2001; 285:2481-5.

21. Venâncio LS, Burini RC, Yoshida WB. Hiper-homocisteinemia na doença arterial periférica. $J$ Vasc Bras. 2004;3:31-7.

22. Rutherford RB, Baker JD, Erust C, et al. Recommended standards for reports dealing with lower extremity ischemia: revised version. J Vasc Surg. 1997;26:517-38.

23. TASC-The TransAtlantic Inter-Society Consensus. Management of peripheral arterial disease. J Vasc Interv Radiol 2003; $14:$ S351.

24. World Health Organization. Obesity: preventing and managing the global epidemic. Geneva: WHO; 2003.

25. Sociedade Brasileira de Cardiologia, Departamento de Aterosclerose. IV Diretriz Brasileira sobre Dislipidemia e Prevenção da Atrosclerose. Arq Bras Cardiol. 2007;88(supl 1).

26. Araki A, Sako Y. Determination of free and total homocysteine in human plasma by high-performance liquid chromatography with fluorescence detection. J Chromatogr. 1987;422:43-52.

27. Ubbink JB, Vermaak WJ, Bissbort S. Rapid high performance chromatographic assay for total homocysteine levels in human serum. J Chromatogr. 1991;565:441-6.

28. Ueland PM, Refsum H, Stabler SP, Malinow MR, Andersson A, Allen RH. Total homocysteine in plasma or serum: methods and clinical applications. Clin Chem. 1993; 39:1764-79.

29. Van Den Bosch MA, Bloemenkamp DG, Mali WP. Hyperhomocysteinemia and risk for peripheral arterial occlusive disease in young women. J Vasc Surg. 2003;38:772-8.

30. Bree A, Verschuren WM, Blom HJ, Nadeau M, Trijbels FJ, Kromhout D. Coronary heart disease mortality, plasma homocysteine, and B-vitamins: a prospective study. Atherosclerosis. 2003;166:369-77.

31. Rassoul F, Richter V, Janke C, et al. Plasma homocysteine and lipoprotein profile in patients with peripheral arterial occlusive disease. Angiology. 2000;51:189-96.

32. Taute BM, Taute R, Heins S, Behrmann C, Podhaisky S. Hyperhomocisteinemia: marker of sistemic atherosclerosis in peripheral arterial disease. Int Angiol. 2004;23:35-40.

33. Baen LS, Zorn WG, van bellen B. Homocysteine in abdominal aortic aneurysm and occlusive disease. An Paul Med Cir. 2003;130:68-70.

34. Garófolo L, Barros Jr N, Miranda Jr F, D'Almeida V, Cardien LC, Ferreira SR. Association of increased levels of homocysteine and peripheral arterial disease in a JapaneseBrazilian population. Eur J Vasc Endovasc Surg. 2007;34:23-8.

35. Nygard O, Vollset SE, Refsum H, et al. Total plasma homocysteine and cardiovascular risk profile. The Hordaland homocysteine study. JAMA. 1995;274:1526-33.

36. Jacques PF, Bostom AG, Wilson PW, Rosenberg IH, Selhub J. Determinants of plasma total homocysteine concentration in the Framingham offspring cohort. Am J Clin Nutr. 2001;73:613-21.

37. Fukagawa NK, Martin JM, Wurthmann A, Prue AH, Ebenstein D, O'rourke B. Sex-related differences in methionine metabolism and plasma homocysteine concentrations. Am J Clin Nutr. 2000;72:22-9. 
38. Elhadd TA, Neary R, Abdu TA, et al. Influence of the hormonal changes during the normal menstrual cycle in healthy young women on soluble adhesion molecules, plasma homocysteine, free radical markers and lipoprotein fractions. Int Angiol. 2003;22:222-8.

39. Hernanz A. Changes in the intracellular homocysteine and glutathione content associated with aging. Life Sci. 2000;67:1317-24.

40. Clarke R, Frost C, Leroy V, Collins R. Lowering blood homocysteine with folic acid based supplements: metaanalysis of randomized trials. BMJ. 1998;316:894-8.

41. Mc Cully KS. Homocysteine, vitamins, and vascular disease prevention. Am J Clin Nutr. 2007;86:1563S-8S.

Correspondência:

Luciene de Souza Venâncio

Av. Floriano Peixoto, 240

Caixa Postal 334

CEP 18603-970 - Botucatu, SP

E-mail: lucienenutri@yahoo.com.br

\section{Contribuições dos autores}

Concepção e desenho do estudo: LSV, RCB, WBY

Análise e interpretação dos dados: LSV, WBY

Coleta de dados: LSV

Redação do artigo: LSV, WBY

Revisão crítica do texto: LSV, WBY

Aprovação final do artigo*: LSV, RCB, WBY

Análise estatística: LSV

Responsabilidade geral pelo estudo: LSV

Informações sobre financiamento: CAPES e FAPESP

*Todos os autores leram e aprovaram a versão final submetida ao J Vasc Bras. 